\title{
A refinement of the right-hand side of the Hermite-Hadamard inequality for simplices
}

\author{
Monika Nowicka And Alfred Witkowski[
}

\begin{abstract}
We establish a new refinement of the right-hand side of the Hermite-Hadamard inequality for simplices, based on the average values of a convex function over the faces of a simplex and over the values at their barycenters.
\end{abstract}

Mathematics Subject Classification. Primary 26D15.

Keywords. Hermite-Hadamard inequality, Convex function, Simplex, Barycentric coordinates.

\section{Introduction}

The classical Hermite-Hadamard inequality [2] states that for a convex function $f:[a, b] \rightarrow \mathbb{R}$

$$
f\left(\frac{a+b}{2}\right) \leq \frac{1}{b-a} \int_{a}^{b} f(t) \mathrm{d} t \leq \frac{f(a)+f(b)}{2} .
$$

There are many generalizations. One of them $([1,5,6])$ says that if $\Delta \subset \mathbb{R}^{n}$ is a simplex with barycenter $\mathbf{b}$ and vertices $\mathbf{x}_{0}, \ldots, \mathbf{x}_{n}$ and $f: \Delta \rightarrow \mathbb{R}$ is convex, then

$$
f(\mathbf{b}) \leq \frac{1}{\operatorname{Vol}(\Delta)} \int_{\Delta} f(\mathbf{x}) \mathrm{d} \mathbf{x} \leq \frac{f\left(\mathbf{x}_{0}\right)+\cdots+f\left(\mathbf{x}_{n}\right)}{n+1} .
$$

In this paper we aim to improve the right-hand side of the last inequality.

Our main result can be formulated as follows: suppose we split the vertices of a simplex $\Delta$ into disjoint sets $K_{i}, i=1, \ldots, p$ and denote by $\Delta_{K_{i}}$ the face

This work was completed with the support of our $\mathrm{T}_{\mathrm{E} X} \mathrm{X}$-pert.

Birkhäuser 
of $\Delta$ with vertices in $K_{i}$. Then for every convex function $f$ defined on $\Delta$ we have

$$
\operatorname{Avg}(f, \Delta) \leq \sum_{i=1}^{p} \frac{\operatorname{dim} \Delta_{K_{i}}+1}{\operatorname{dim} \Delta+1} \operatorname{Avg}\left(f, \Delta_{K_{i}}\right),
$$

where $\operatorname{Avg}(f, \Delta)$ denotes the average value of $f$ on the simplex with respect to an appropriate Lebesgue measure. We analyze the relationship between different right-hand sides of (3).

We also provide a class of refinements involving the values of the function $f$ at barycenters of faces.

\section{Definitions and lemmas}

For a fixed natural number $n \geq 1$ let $N=\{0,1, \ldots, n\}$. Suppose $\mathbf{x}_{0}, \ldots, \mathbf{x}_{n} \in$ $\mathbb{R}^{n}$ are such that the vectors $\overrightarrow{\mathbf{x}_{0} \mathbf{x}_{i}}, i=1, \ldots, n$ are linearly independent.

Suppose $K$ is a nonempty subset of $N$ with $k+1$ elements $(0 \leq k \leq$ $n)$ and denote the elements of $\left\{\mathbf{x}_{i}: i \in K\right\}$ by $\left\{\mathbf{y}_{0}, \ldots, \mathbf{y}_{k}\right\}$. The set $\Delta_{K}=$ $\operatorname{conv}\left\{\mathbf{y}_{0}, \ldots, \mathbf{y}_{k}\right\}$ is called a simplex (or a $k$-simplex if we want to emphasize its dimension). The $n$-simplex $\Delta_{N}$ will be denoted by $\Delta$.

Given two sets $K \subset L \subset N$ the simplex $\Delta_{K}$ is called a face (or $k$-face) of $\Delta_{L}$.

The points in $\Delta_{K}$ admit a unique representation of the form

$$
\mathbf{y}=\sum_{i=0}^{k} \alpha_{i} \mathbf{y}_{i}, \quad \alpha_{i} \geq 0, \quad \sum_{i=0}^{k} \alpha_{i}=1 .
$$

The $k+1$-tuple $\left(\alpha_{0}, \ldots, \alpha_{k}\right)$ is called barycentric coordinates. The point

$$
\mathbf{b}_{K}=\frac{1}{k+1}\left(\mathbf{y}_{0}+\cdots+\mathbf{y}_{k}\right)
$$

is called the barycenter of $\Delta_{K}$.

If $\Sigma$ is a $k$-dimensional simplex and $f: \Sigma \rightarrow \mathbb{R}$ is an integrable function, then we shall denote its average value over the simplex by $\operatorname{Avg}(f, \Sigma)$, i.e.

$$
\operatorname{Avg}(f, \Sigma)=\frac{1}{\operatorname{Vol}(\Sigma)} \int_{\Sigma} f(\mathbf{x}) \mathrm{d} \mathbf{x}
$$

The integration here is with respect to the $k$-dimensional Lebesgue measure denoted by $\mathrm{d} \mathbf{x}$ and $\operatorname{Vol}(\Sigma)$ denotes the $k$-dimensional volume.

With this notation we can write the right-hand side of (2) as

$$
\operatorname{Avg}(f, \Delta) \leq \frac{1}{n+1} \sum_{i=0}^{n} \operatorname{Avg}\left(f, \Delta_{\{i\}}\right) .
$$

The set $E_{k}=\left\{\left(\alpha_{1}, \ldots, \alpha_{k}\right): \alpha_{i} \geq 0, \sum_{i=1}^{k} \alpha_{i} \leq 1\right\} \subset \mathbb{R}^{k}$ is called a standard simplex. 
For every $k$-simplex $\Delta_{K}$ we define a one-to-one mapping $\varphi_{K}: E_{k} \rightarrow \Delta_{K}$ given by the formula

$$
\varphi_{K}\left(\alpha_{1}, \ldots, \alpha_{k}\right)=\mathbf{y}_{0}+\sum_{i=1}^{k} \alpha_{i}\left(\mathbf{y}_{i}-\mathbf{y}_{0}\right) .
$$

Note that $\partial \varphi_{K} / \partial \alpha_{i}=\mathbf{y}_{i}-\mathbf{y}_{0}$, so the absolute value of its Jacobian equals $\left|\operatorname{det} D \varphi_{K}\right|=k ! \operatorname{Vol}\left(\Delta_{K}\right)$. This means, that if $g: \Delta_{K} \rightarrow \mathbb{R}$ is an integrable function, then the identity

$$
\frac{1}{\operatorname{Vol}\left(\Delta_{K}\right)} \int_{\Delta_{K}} g(\mathbf{x}) \mathrm{d} \mathbf{x}=k ! \int_{E_{k}} g\left(\varphi_{K}(\boldsymbol{\alpha})\right) \mathrm{d} \boldsymbol{\alpha}
$$

holds. In particular $\operatorname{Vol}\left(E_{k}\right)=1 / k$ !

A partition of $N$ is a set

$$
\mathcal{K}=\left\{K_{i}: i=1 \ldots p, K_{i} \subset N, \bigcup_{i=1}^{p} K_{i}=N, K_{i} \cap K_{j}=\emptyset\right\} .
$$

We say that $\mathcal{L}$ refines $\mathcal{K}$ (and write $\mathcal{L} \prec \mathcal{K}$ ) if every element of $\mathcal{L}$ is a subset of an element of $\mathcal{K}$.

\section{Faces based refinements}

The inequality (4) shows that the average of a convex function over a simplex can be bounded by the convex combination of its average values over its 0 -faces. Our main result-Theorem 3.1 and its corollaries generalize this fact.

Theorem 3.1. Let $\mathcal{K}=\left\{K_{1}, \ldots, K_{p}\right\}$ be a partition of $N$ and $f: \Delta \rightarrow \mathbb{R}$ be a convex function. Define

$$
F(\mathcal{K})=\sum_{i=1}^{p} \operatorname{card} K_{i} \operatorname{Avg}\left(f, \Delta_{K_{i}}\right) .
$$

If $\mathcal{L} \prec \mathcal{K}$, then

$$
F(\mathcal{K}) \leq F(\mathcal{L}) .
$$

To prove this theorem we shall need Lemma 3.2.

Lemma 3.2. Let $K, L \subset N$ be two disjoint, nonempty sets with $\operatorname{card} K=k+1$ and $\operatorname{card} L=l+1$. Further, let $f: \Delta \rightarrow \mathbb{R}$ be a convex function. Then

$$
\operatorname{Avg}\left(f, \Delta_{K \cup L}\right) \leq \frac{k+1}{k+l+2} \cdot \operatorname{Avg}\left(f, \Delta_{K}\right)+\frac{l+1}{k+l+2} \cdot \operatorname{Avg}\left(f, \Delta_{L}\right) .
$$


Proof. Denote by $\mathbf{u}_{0}, \ldots, \mathbf{u}_{k}$ the vertices of $\Delta_{K}$ and by $\left(\alpha_{0}, \ldots, \alpha_{k}\right)$ its barycentric coordinates. Similarly, let $\mathbf{v}_{0}, \ldots, \mathbf{v}_{l}$ be the vertices of $\Delta_{L}$ and $\left(\beta_{0}, \ldots, \beta_{l}\right)$ be its barycentric coordinates. Every point $\mathbf{x} \in \Delta_{K \cup L}$ can be represented as

$$
\mathbf{x}=\sum_{i=0}^{k} \alpha_{i}^{\prime} \mathbf{u}_{i}+\sum_{j=0}^{l} \beta_{j}^{\prime} \mathbf{v}_{j}, \alpha_{i}^{\prime}, \beta_{j}^{\prime} \geq 0, \sum_{i=0}^{k} \alpha_{i}^{\prime}+\sum_{j=0}^{l} \beta_{j}^{\prime}=1 .
$$

Let $\sum_{i=0}^{k} \alpha_{i}^{\prime}=s, \sum_{j=0}^{l} \beta_{j}^{\prime}=1-s$ and $\alpha_{i}=\alpha_{i}^{\prime} / s, \beta_{j}=\beta_{j}^{\prime} /(1-s)$ (if division by zero occurs we assume the result is zero). Then

$$
\mathbf{x}=s \sum_{i=0}^{k} \alpha_{i} \mathbf{u}_{i}+(1-s) \sum_{j=0}^{l} \beta_{j} \mathbf{v}_{j} .
$$

Let $\Phi:[0,1] \times E_{k} \times E_{l} \rightarrow \Delta_{K \cup L}$ be defined by

$$
\begin{aligned}
& \Phi\left(s, \alpha_{1}, \ldots, \alpha_{k}, \beta_{1}, \ldots, \beta_{l}\right) \\
& \quad=s\left(\mathbf{u}_{0}+\sum_{i=1}^{k} \alpha_{i}\left(\mathbf{u}_{i}-\mathbf{u}_{0}\right)\right)+(1-s)\left(\mathbf{v}_{0}+\sum_{j=1}^{l} \beta_{j}\left(\mathbf{v}_{j}-\mathbf{v}_{0}\right)\right) \\
& \quad=s \varphi_{K}\left(\alpha_{1}, \ldots, \alpha_{k}\right)+(1-s) \varphi_{L}\left(\beta_{1}, \ldots, \beta_{l}\right) .
\end{aligned}
$$

Its Jacobian equals

$$
\begin{aligned}
\operatorname{det} D \Phi & =\left|\begin{array}{c}
\Phi_{s}^{\prime} \\
\vdots \\
\Phi_{\alpha_{i}}^{\prime} \\
\vdots \\
\Phi_{\beta_{j}}^{\prime} \\
\vdots
\end{array}\right|=\left|\begin{array}{c}
\mathbf{u}_{0}-\mathbf{v}_{0}+\sum_{i=1}^{k} \alpha_{i}\left(\mathbf{u}_{i}-\mathbf{u}_{0}\right)-\sum_{j=1}^{l} \beta_{j}\left(\mathbf{v}_{j}-\mathbf{v}_{0}\right) \\
\vdots \\
s\left(\mathbf{u}_{i}-\mathbf{u}_{0}\right) \\
\vdots \\
(1-s)\left(\mathbf{v}_{j}-\mathbf{v}_{0}\right) \\
\vdots
\end{array}\right| \\
& =s^{k}(1-s)^{l}\left|\begin{array}{c}
\mathbf{u}_{0}-\mathbf{v}_{0} \\
\vdots \\
\mathbf{u}_{0}-\mathbf{v}_{0} \\
\vdots \\
\mathbf{u}_{i}-\mathbf{u}_{0} \\
\vdots \\
\mathbf{v}_{j}-\mathbf{v}_{0} \\
\vdots
\end{array}\right|=s^{k}(1-s)^{l}\left|\begin{array}{c}
\mathbf{v}_{0} \\
\vdots \\
\mathbf{v}_{j}-\mathbf{v}_{0} \\
\vdots \\
\vdots
\end{array}\right|
\end{aligned}
$$

Changing the variable in the integral yields

$$
\int_{\Delta_{K} \cup L} f(\mathbf{x}) \mathrm{d} \mathbf{x}=\int_{0}^{1} \mathrm{~d} s \int_{E_{k}} \mathrm{~d} \boldsymbol{\alpha} \int_{E_{l}} \mathrm{~d} \boldsymbol{\beta} f(\Phi(s, \boldsymbol{\alpha}, \boldsymbol{\beta}))|\operatorname{det} D \Phi| .
$$


Using the convexity of $f$ and the formula (5) we obtain

$$
\begin{aligned}
\int_{0}^{1} \mathrm{~d} s & \int_{E_{k}} \mathrm{~d} \boldsymbol{\alpha} \int_{E_{l}} \mathrm{~d} \boldsymbol{\beta} f(\Phi(s, \boldsymbol{\alpha}, \boldsymbol{\beta})) s^{k}(1-s)^{l} \\
= & \int_{0}^{1} s^{k}(1-s)^{l} \mathrm{~d} s \int_{E_{k}} \mathrm{~d} \boldsymbol{\alpha} \int_{E_{l}} \mathrm{~d} \boldsymbol{\beta} f\left(s \varphi_{K}(\boldsymbol{\alpha})+(1-s) \varphi_{L}(\boldsymbol{\beta})\right) \\
\leq & \int_{0}^{1} s^{k+1}(1-s)^{l} \mathrm{~d} s \int_{E_{k}} f\left(\varphi_{K}(\boldsymbol{\alpha})\right) \mathrm{d} \boldsymbol{\alpha} \int_{E_{l}} \mathrm{~d} \boldsymbol{\beta} \\
& +\int_{0}^{1} s^{k}(1-s)^{l+1} \mathrm{~d} s \int_{E_{k}} \mathrm{~d} \boldsymbol{\alpha} \int_{E_{l}} f\left(\varphi_{L}(\boldsymbol{\beta})\right) \mathrm{d} \boldsymbol{\beta} \\
= & \frac{(k+1) ! l !}{(k+l+2) !} \cdot \frac{\operatorname{Avg}\left(f, \Delta_{K}\right)}{k !} \cdot \frac{1}{l !}+\frac{k !(l+1) !}{(k+l+2) !} \cdot \frac{1}{k !} \cdot \frac{\operatorname{Avg}\left(f, \Delta_{L}\right)}{l !} .
\end{aligned}
$$

The Lemma follows from (7), (8) and (9).

Now we can prove our main result.

Proof of Theorem 3.1. It follows from Lemma 3.2 that the function

$$
K \mapsto \operatorname{card} K \operatorname{Avg}\left(f, \Delta_{K}\right)
$$

is subadditive on disjoint sets, so (6) follows by mathematical induction on the cardinality of partition.

Since $\{\{0\}, \ldots,\{n\}\} \prec \mathcal{K} \prec\{N\}$ for any partition $\mathcal{K}$, we obtain the refinements of the Hermite-Hadamard inequality.

Corollary 3.3. For any partition $\mathcal{K}$ the inequalities

$$
(n+1) \operatorname{Avg}(f, \Delta) \leq F(\mathcal{K}) \leq f\left(\mathbf{x}_{0}\right)+\cdots+f\left(\mathbf{x}_{n}\right)
$$

hold.

Applying Corollary 3.3 to all possible pairs consisting of a vertex and its opposite face one obtains the following result.

Corollary 3.4. Let $f: \Delta \rightarrow \mathbb{R}$ be a convex function. Then the inequality

$$
\begin{aligned}
\operatorname{Avg}(f, \Delta) \leq & \frac{1}{n+1} \frac{f\left(\mathbf{x}_{0}\right)+\cdots+f\left(\mathbf{x}_{n}\right)}{n+1} \\
& +\frac{n}{n+1} \cdot \frac{1}{n+1} \sum_{\substack{K \subset N \\
\operatorname{card} K=n}} \operatorname{Avg}\left(f, \Delta_{K}\right)
\end{aligned}
$$

holds.

If $N$ can be divided into disjoint subsets of the same cardinality, then applying Corollary 3.3 to all possible partitions and summing the obtained inequalities one gets the following corollary. 
Corollary 3.5. Let $f: \Delta \rightarrow \mathbb{R}$ be a convex function and $d$ be a divisor of $n+1=\operatorname{card} N$. Then

$$
\operatorname{Avg}(f, \Delta) \leq \frac{1}{\left(\begin{array}{c}
n+1 \\
d
\end{array}\right)} \sum_{\substack{K \subset N \\
\operatorname{card} K=d}} \operatorname{Avg}\left(f, \Delta_{K}\right) .
$$

It is known (see e.g. [7, p. 125]) that the volume of a regular simplex $\Delta \subset \mathbb{R}^{n}$ with unit edges equals $\frac{1}{n !} \sqrt{\frac{n+1}{2^{n}}}$. In this case Corollary 3.5 yields

Corollary 3.6. Let $f$ be a convex function defined on a regular simplex $\Delta$ with unit edges. Let $d$ be a divisor of $n+1=\operatorname{card} N$. Then

$$
\begin{aligned}
& \int_{\Delta} f(\mathbf{x}) \mathrm{d} \mathbf{x} \\
& \leq\left[\left(\begin{array}{c}
n \\
d-1
\end{array}\right)\right]^{-2} \frac{1}{(n+1-d) !} \sqrt{\frac{d}{n+1} \frac{1}{2^{n+1-d}}} \sum_{\substack{K \subset N \\
\operatorname{card} K=d}} \int_{\Delta_{K}} f(\mathbf{x}) \mathrm{d} \mathbf{x} .
\end{aligned}
$$

\section{Barycentric refinements}

It is known that the right-hand side of the Hermite-Hadamard inequality can be refined as follows $([3,4,8])$ :

$$
\frac{1}{b-a} \int_{a}^{b} f(t) \mathrm{d} t \leq \frac{1}{2}\left[f\left(\frac{a+b}{2}\right)+\frac{f(a)+f(b)}{2}\right],
$$

or in the case of a simplex

$$
\operatorname{Avg}(f, \Delta) \leq \frac{1}{n+1} f(\mathbf{b})+\frac{n}{n+1} \frac{f\left(\mathbf{x}_{0}\right)+\cdots+f\left(\mathbf{x}_{n}\right)}{n+1} .
$$

Given the fact that the vertices are in fact the barycenters of 0 -faces, we see that in this case the average value of $f$ over a simplex is bounded by a convex combination of its values at the barycenters of all faces.

The next theorem improves (10) and can be a base for further refinements.

Theorem 4.1. Let $\mathbf{b}$ be the barycenter of $\Delta$ and let $K_{i}=N \backslash\{i\}$. If $f: \Delta \rightarrow \mathbb{R}$ is convex, then

$$
\operatorname{Avg}(f, \Delta) \leq \frac{1}{n+1} f(\mathbf{b})+\frac{n}{n+1} \cdot \frac{1}{n+1} \sum_{i=0}^{n} \operatorname{Avg}\left(f, \Delta_{K_{i}}\right) .
$$

Proof. Note that we can identify the partitions of $N$ with partitions of the set of vertices of a simplex. The barycenter divides $\Delta$ into $n+1$ simplices

$$
B_{i}=\operatorname{conv}\left\{\mathbf{x}_{0}, \ldots, \mathbf{x}_{i-1}, \mathbf{b}, \mathbf{x}_{i+1}, \ldots, \mathbf{x}_{n}\right\}, \quad i=0, \ldots, n .
$$


For each of them we split its vertices into two groups: $\{\mathbf{b}\}$ and $\left\{\mathbf{x}_{j}: j \neq i\right\}$ and apply Lemma 3.2 to these partitions. Taking into account that $\operatorname{Vol}\left(B_{i}\right)=$ $\frac{1}{n+1} \operatorname{Vol}(\Delta)$ for every $i$, we get

$$
\begin{aligned}
\frac{\int_{B_{i}} f(\mathbf{x}) \mathrm{d} \mathbf{x}}{\operatorname{Vol}(\Delta)} & =\frac{1}{n+1} \operatorname{Avg}\left(f, B_{i}\right) \\
& \leq \frac{1}{n+1}\left[\frac{1}{n+1} f(\mathbf{b})+\frac{n}{n+1} \operatorname{Avg}\left(f, \Delta_{K_{i}}\right)\right]
\end{aligned}
$$

Summing these inequalities completes the proof.

Now one can see that applying (2) to all simplices in (11) we obtain (10). But we can do much better: instead of (2) we can use Lemma 4.1 recursively to $(n-1)$-faces etc. and continue this process until we reach 0 -faces. Thus we obtain

Theorem 4.2. Under the assumptions of Theorem 4.1

$$
\operatorname{Avg}(f, \Delta) \leq \frac{1}{n+1} \sum_{k=1}^{n+1} \frac{1}{\left(\begin{array}{c}
n+1 \\
k
\end{array}\right)} \sum_{\substack{K \subset N \\
\operatorname{card} K=k}} f\left(\mathbf{b}_{K}\right)
$$

Combining the results of Corollary 3.3 and Theorem 4.1 one can produce various new upper bounds for the average value of $f$ over the simplex. Below we show one of them.

Splitting the vertices of $\Delta$ into the maximum number of groups of $k$ elements and one group of $l$ elements, where $l<k$, and taking the average over all such splits one gets

Corollary 4.3. Let $\Delta \subset \mathbb{R}^{n}$ be an arbitrary simplex and let $\mathbf{x}_{0}, \ldots, \mathbf{x}_{n}$ be its vertices.

Then for every convex function $f: \Delta \rightarrow \mathbb{R}$ we have

$$
\operatorname{Avg}(f, \Delta) \leq \alpha_{n} \frac{1}{\left(\begin{array}{c}
n+1 \\
k
\end{array}\right)} \sum_{\substack{K \subset N \\
\operatorname{card} K=k}} f\left(\mathbf{b}_{K}\right)+\left(1-\alpha_{n}\right) \frac{f\left(\mathbf{x}_{0}\right)+\cdots+f\left(\mathbf{x}_{n}\right)}{n+1}
$$

where $\alpha_{n}=\frac{\left\lfloor\frac{n+1}{k}\right\rfloor}{n+1}$.

In particular we obtain this corollary.

Corollary 4.4. Let $\Delta \subset \mathbb{R}^{n}$ be an arbitrary simplex with vertices $\mathbf{x}_{0}, \ldots, \mathbf{x}_{n}$. Then for every convex function $f: \Delta \rightarrow \mathbb{R}$ we have

$$
\operatorname{Avg}(f, \Delta) \leq \alpha_{n} \frac{1}{\left(\begin{array}{c}
n+1 \\
2
\end{array}\right)} \sum_{0 \leq i<j \leq n} f\left(\frac{\mathbf{x}_{i}+\mathbf{x}_{j}}{2}\right)+\beta_{n} \frac{f\left(\mathbf{x}_{0}\right)+\cdots+f\left(\mathbf{x}_{n}\right)}{n+1},
$$

where $\alpha_{n}=\frac{\left\lfloor\frac{n+1}{2}\right\rfloor}{n+1}$ and $\beta_{n}=\frac{\left\lceil\frac{n+1}{2}\right\rceil}{n+1}$. 
Open Access. This article is distributed under the terms of the Creative Commons Attribution 4.0 International License (http://creativecommons.org/licenses/by/4.0/), which permits unrestricted use, distribution, and reproduction in any medium, provided you give appropriate credit to the original author(s) and the source, provide a link to the Creative Commons license, and indicate if changes were made.

\section{References}

[1] Bessenyei, M.: The Hermite-Hadamard inequality on simplices. Am. Math. Mon. 115(4), 339-345 (2008)

[2] Hadamard, J.: Étude sur les propriétés des fonctions entières et en particulier d'une fonction considérée par Riemann. J. Math. Pures Appl. 58, 171-215 (1893)

[3] Hammer, P.C.: The midpoint method of numerical integration. Math. Mag. 31, 193-195 $(1957 / 1958)$

[4] Mitroi, F.-C., Spiridon, C.I.: Refinements of Hermite-Hadamard inequality on simplices. Math. Rep. (Bucur.) 15(65(1)), 69-78 (2013)

[5] Neuman, E.: Inequalities involving multivariate convex functions II. Proc. Am. Math. Soc. 109(4), 965-974 (1990)

[6] Niculescu, C.P.: The Hermite-Hadamard inequality for convex functions of a vector variable. Math. Inequal. Appl. 5(4), 619-623 (2002)

[7] Sommerville, D.M.Y.: An Introduction to the Geometry of $n$ Dimensions. Dover Publications. Inc., New York (1958)

[8] Wąsowicz, S., Witkowski, A.: On some inequality of Hermite-Hadamard type. Opuscula Math. 32(3), 591-600 (2012)

Monika Nowicka

Institute of Mathematics and Physics

UTP University of Science and Technology

al. prof. Kaliskiego 7,

85-796 Bydgoszcz,

Poland

e-mail: monika.nowicka@utp.edu.pl

Alfred Witkowski

Institute of Mathematics and Physics

UTP University of Science and Technology

al. prof. Kaliskiego 7,

85-796 Bydgoszcz,

Poland

e-mail: alfred.witkowski@utp.edu.pl

Received: May 27, 2016 